\section{Opening new windows in observing the}

\section{Universe}

\author{
G.-A. Tammann, E-K. Thielemann, D. Trautmann \\ Dept. of Physics and Astronomy, Univ. of Basel
}

The 2002 Nobel prizes in Physics underline the close relation$I$ ship between physics and astronomy in understanding the Universe and its stellar constituents via novel detection technics like giant underground particle detectors or space born X-ray telescopes. Raymond Davis (retired from the University of Pennsylvania) and Masotoshi Koshiba (retired from the University of Tokyo) obtained one half of the prize for discovering and detecting neutrinos from the sun or respectively from supernova explosions. The other half of the prize went to Roberto Giacconi (Director of Associated Universities Inc. in Washington) for the discovery of cosmic X-ray sources. As X-rays cannot penetrate the Earth's atmosphere, their detection was only possible via rocket or balloon flights (or later with satellites). In all three cases pioneers were honored who opened new windows in observing the Universe (www.nobel.se/physics/laureates/2002) and founded vastly expanding research fields leading to additional exciting discoveries.

\section{Cosmic Neutrinos}

Neutrinos were postulated by Pauli in 1930 as essentially massless particles without charge and with a spin $1 / 2$, in order to permit the conservation of energy, charge and angular momentum in nuclear beta-decay $(Z, N) \rightarrow(Z \pm 1, N \bar{\mp})+e^{-}\left(e^{+}\right)+\bar{v}_{e}\left(v_{e}\right)$, where $Z$ and $N$ stand for the number of protons $(\mathrm{p})$ and neutrons $(\mathrm{n})$ in a nucleus, $e^{\mp}$ for an electron (or a positron), $\bar{v}_{\varepsilon}$ for an (electron-) antineutrino and $v_{e}$ for a neutrino. This decay mode is equivalent to neutrino (antineutrino) or electron (positron) capture when moving these particles from the right side of the reaction equation to the left in form of their antiparticles (and assuring energy/mass conservation). Antineutrinos, originating from nuclear reactors, were discovered in 1955 by Reines and Cowan via $\bar{v}_{e}+p n+e^{+}$, which corresponds to the reaction for $Z=1$ and $N=0$.

\section{The Lack of Solar Neutrinos}

Davis, with a Ph.D. in physical chemistry from Yale, spent almost his entire scientific life at Brookaven National Laboratory. He specialized in radiochemical detection methods, concentrating on low level technics and background reduction. This was an excellent preparation for his plan to detect neutrinos produced in hydrogen burning deep in the solar interior from where the weakly interacting neutrinos escape (almost) freely. In the 1960 s a good basic understanding of stellar evolution and solar hydrogen burning had emerged, based on the reaction cycles proposed by Bethe and von Weizsäcker and many cross section measurements made at Caltech by W. Fowler's group. The pp-cycles which dominate hydrogen burning in the sun, with a net result of $4^{1} \mathrm{H} \rightarrow{ }^{4} \mathrm{He}+2 e^{+}+2 v_{e}$, produce neutrinos in four different reactions, shown in Table 1 for temperatures of roughly $1.5 \times 10^{7} \mathrm{~K}$.
Table 1: Neutrino Production in Solar Hydrogen Burning

\begin{tabular}{|c|c|c|c|c|}
\hline \multicolumn{3}{|c|}{ Reaction } & $\frac{\text { rel. } \nu_{\varepsilon} \text {-emission }}{100}$ & $\frac{E_{\nu_{i}}(\mathrm{MeV})}{<0.420}$ \\
\hline $\begin{array}{r}(\mathrm{pp}){ }^{1} \mathrm{H}+{ }^{1} \mathrm{H} \\
\text { (pep) }{ }^{1} \mathrm{H}+e^{-}+{ }^{1} \mathrm{H} \\
{ }^{7} \mathrm{Be}+e^{-} \\
{ }^{8} \mathrm{~B}\end{array}$ & $\begin{array}{l}\rightarrow \\
\rightarrow \\
\rightarrow\end{array}$ & $\begin{array}{c}{ }^{2} \mathrm{H}+e^{+}+\nu_{e} \\
{ }^{2} \mathrm{H}+\nu_{e} \\
{ }^{7} \mathrm{Li}+\nu_{\epsilon} \\
{ }^{8} \mathrm{Be}^{*}+e^{+}+\nu_{e}\end{array}$ & $\begin{array}{c}100 \\
0.235 \\
8.017 \\
0.009\end{array}$ & $\begin{array}{r}<0.420 \\
1.442 \\
0.861 \\
<15.000\end{array}$ \\
\hline
\end{tabular}

The relative $v_{e}$-emmission rate indicates how many neutrinos are emitted relative to 100 neutrinos from the pp-reaction. Betadecays lead to a distribution of the energy release among neutrinos and positrons, whereas electron captures release the neutrino with the total reaction energy gain. Due to the vanishingly small interaction of neutrinos with matter, it is/was extremely difficult to detect them, although $6 \times 10^{10}$ solar neutrinos penetrate the earth per $\mathrm{cm}^{2}$ and sec. Table 2 lists a number of possible detection reactions, $E_{\text {thr }}$ is the minimum neutrino energy requirement, $\mathrm{cc}$ and nc stand for neutral current or charged current reactions, involving a $Z^{0}$ boson or $W^{\mp}$ bosons.

v Table 1: Neutrino Detection Reactions

\begin{tabular}{rclr}
\hline \hline \multicolumn{3}{c}{ Reaction } & $\mathrm{E}_{t h r}(\mathrm{MeV})$ \\
\hline$\nu_{e}+{ }^{37} \mathrm{Cl}$ & $\rightarrow$ & ${ }^{37} \mathrm{Ar}+e^{-}(\mathrm{cc})$ & 0.814 \\
$\nu_{e}+{ }^{71} \mathrm{Ga}$ & $\rightarrow$ & ${ }^{71} \mathrm{Ge}+e^{-}(\mathrm{cc})$ & 0.233 \\
$\nu+e^{-}$ & $\rightarrow$ & $\nu^{\prime}+e^{-1}(\mathrm{nc}+\mathrm{cc})$ & $>5.00$ \\
$\nu_{e}+{ }^{2} \mathrm{H}$ & $\rightarrow$ & $2^{1} \mathrm{H}+e^{-}(\mathrm{cc})$ & 1.442 \\
$\nu+{ }^{2} \mathrm{H}$ & $\rightarrow$ & $\nu^{\prime}+{ }^{1} \mathrm{H}+n$ (nc) & 2.224 \\
\hline
\end{tabular}

Raymond Davis used a tank filled with 400000 liters of the cleaning fluid $\mathrm{C}_{2} \mathrm{Cl}_{4}$ (perchloroethylene). About one fourth of the chlorine exists as the isotope ${ }^{37} \mathrm{Cl}$, which permits to detect only neutrinos with energies exceeding $0.814 \mathrm{MeV}$, i.e. essentialy only neutrinos from ${ }^{7} \mathrm{Be}$ and ${ }^{8} \mathrm{~B}$ decay. The location at the Homestake Gold Mine in South Dakota, $1.5 \mathrm{~km}$ under ground, permitted to exclude cosmic ray reactions. Radio chemistry methods allowed to extract the ${ }^{37} \mathrm{Ar}$ noble gas atoms and detect their decay with a half-life of 35 days. This experiment ran from $1967[1,2]$ until 1994. On average about 0.45 decays (i.e. neutrinos) per day were detected, a result of the right order of magnitude, but only amounting to about $34 \%$ of the expected flux predicted by the "standard solar model". With this extremely precise radiochemical experiment Davis and his collaborators revealed the "solar neutrino problem" which waited for its solution about 36 years.

\section{The Detection of Supernova Neutrinos}

Masotoshi Koshiba had a background as cosmic-ray and experimental particle physicist and close connections to the University of Chicago, CERN in Geneva and DESY in Hamburg. In the Kamioka zinc mine he built the Nucleon Decay Experiment (KamiokaNDE). It was based on the detection of Cerenkov light emitted by energetic charged decay particles from nucleon decay (or e.g. energetic electrons scattered by such particles) moving with velocities larger than the speed of light in the medium water. This leads, similar to sonic booms, to radiation emitted in a narrow forward cone along the incident direction of the moving particle. The resulting light flashes can be detected with an array of photo multiplier tubes. Early versions of Grand Unified Theories (GUTs), unifying strong, weak and electromagnetic forces had predicted decay half-lives of the "stable" proton of the order $10^{30}$ years. The total amount of protons in 3000 tons of water would have permitted to observe a sufficient number of decays, the non-detection provided only lower limits for the half-life. Koshiba's achievement was to adapt KamiokaNDE in 1986 to a 


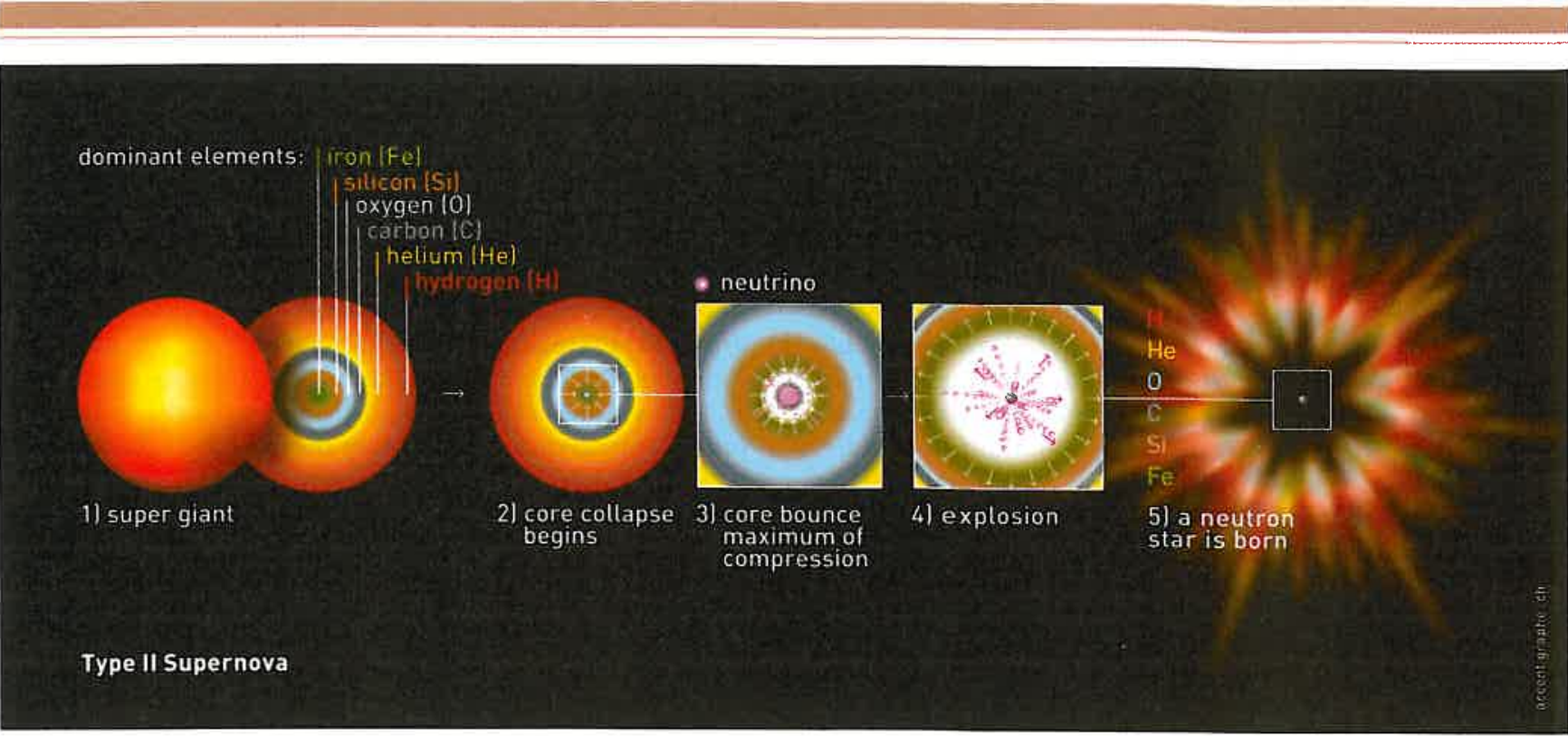

A Fig. 2: The nearby galaxy NGC 4697 as seen by the Chandra Xray observatory. The hot gas in the galaxy radiates only weakly. The bright spots are black holes and neutron stars, accreting gas from a binary companion star

(http://chandra.harvard.edu/photo/2002/1140/).

Combined with other efforts, e.g. the European X-ray satellites ROSAT, BeppoSAX and XMM-Newton, the Russian Granat and MIR-Kvant missions, and the Japanese satellites Ginga and ASCA, this led to a wealth of discoveries, related to different types of Xray radiation from astrophysical sources. These include (i) thermal black body radiation with temperatures corresponding to $\mathrm{X}$-ray energies, (ii) synchrotron radiation from electrons moving in strong magnetic fields, and (iii) X-ray line emission from excited atoms. A wealth of phenomena and objects have been observed with XMM [9].

$\mathrm{X}$-ray line emission from a hot gas can be observed in supernova remnants (revealing their element composition) or in clusters of galaxies [10]. Compact objects like white dwarfs, neutron stars or black holes in binary stellar systems can accrete matter into their deep gravitational potential holes from the binary companion star, leading to a hot gas which emits thermal $\mathrm{X}$-rays or in some cases also to the ignition of thermonuclear burning (type I X-ray bursts). Figure 2 shows a Chandra image of such compact X-ray sources in the galaxy NGC 4797. Massaccreting black holes emit X-rays and in some cases jets, relativistic electrons in these jets emit synchrotron radiation. Supermassive black holes (of the order of $10^{6}$ solar masses) in the center of galaxies cause "active galactic nuclei". In some cases their jets extend over millions of light years. The (initially) apparently isotropic X-ray background can now be resolved into individual sources of active galaxies with distances up to and exceeding 12 billion light years (visible with XMM and Chandra).

The study of this wealth of phenomena and objects, visible in the X-ray range, became possible after the pioneering efforts of $R$. Giacconi. He combines the qualities of a scientist and a manager in an exellent way, and served the astronomical community also as director of the Space Telescope Science Institute in Baltimore, director of the European Southern Observatory (ESO), and since 1999 as director of American Universities Inc. which builds together with ESO a giant telesope for cosmic infrared radiation in Chile.

\section{References}

[1] Davis, R., Harmer, D.S., Hoffman, K.C., Phys. Rev. Lett. 20, 1205 (1968)

[2] Bahcall, J.N., Bahcall, N.E., Shaviv, G., Phys. Rev. Lett. 20, 1209 (1968)

[3] Hirata, K., Kajita, T., Koshiba, M. et al., Phys. Rev. Lett. 58, 1490 (1987)

[4] Kirsten, T., Rev. Mod. Phys. 71, 1213 (1999)

[5] Ahmad, Q.R. et al., Phys. Rev. Lett. 89,011301 (2002)

[6] Eguchi, K., Phys. Rev. Lett. 90, 021802 (2003)

[7] Giacconi, R., Gursky, H., Paolini, F.R., Rossi, B.B., Phys. Rev. Lett. 9, 439 (1962)

[8] Giacconi, R. et al., Astrophys. J. 230, 540 (1979)

[9] Astron. Astrophys. Lett. 365 (2001)

[10] Rosati, P., Borgani, S., Norman, C., Ann. Rev. Astron. Astrophys. 40, 539 (2002)

\section{About the authors}

Gustav A. Tammann studied astronomy in Basel under W. Becker. He came in 1963 to the Mount Wilson and Palomar Observatories (now: Carnegie Observatories) to work with Allan Sandage on the expansion rate and the age of the Universe; the collaboration continues to the present day. From 1977 to 2002 he was professor of astronomy at the University of Basel. Among other honors he received in 2000 the Tomalla Prize for Gravitation and Cosmology.

Friedrich-Karl Thielemann received his $\mathrm{PhD}$ at the Max-PlanckInstitute for Astrophysics in Garching in 1980. After having held a faculty position at Harvard University, he became professor of theoretical physics at the University of Basel in 1994. He is elected fellow of the American Physical Society and Associate Editor of Nuclear Physics A. His research interests include wide areas of nuclear physics and astrophysics.

Dirk Trautmann received his $\mathrm{PhD}$ at the University of Basel in 1969. After the habilitation in 1975 he spent a number of extended visits at universities in Europe, South Africa, Mexico and the US. Since 1987 he is professor for theoretical physics at the University of Basel. His research interests are related to atomic and nuclear reactions with heavy ions and cover many topics in atomic, nuclear and particle physics. 